\title{
FABRICAÇÃO DE COMPÓSITOS DE MATRIZ POLIÉSTER REFORÇADA COM TECIDO DE JUTA APLICADOS EM BLINDAGEM MULTICAMADA*
}

\author{
Foluke Salgado de Assis ${ }^{1}$ \\ Sergio Neves Monteiro ${ }^{2}$ \\ Felipe Magalhães de Matos Gabrie/ ${ }^{3}$ \\ Daniel Neri Nogueira do Nascimento ${ }^{3}$ \\ Kessy Jhonnes Matheus Marques Magalhães ${ }^{3}$ \\ Daniel Fernandes Ferreira ${ }^{3}$ \\ Amon Rhaniery Brito Machado ${ }^{3}$ \\ Artur Camposo Pereira ${ }^{4}$
}

\section{Resumo}

Este trabalho visa apresentar os resultados obtidos através de ensaios balísticos realizados em sistemas de blindagem multicamada. Seguindo as especificações da norma americana NIJ 0101.06, foram realizados ensaios utilizando munição de calibre 7,62x $51 \mathrm{~mm}$, que possui velocidade acima de $800 \mathrm{~m} / \mathrm{s}$. A blindagem multicamada utilizada possui como camada frontal uma pastilha hexagonal cerâmica (alumina dopada com 4\% de nióbia), segunda camada de compósito de matriz poliéster reforçada com tecido de juta e como terceira camada uma placa de alumino 5052 H34. A utilização de compósitos poliméricos reforçados com fibras naturais em substituição às fibras de aramida é de grande interesse, pois além de desempenharem a mesma função no sistema de blindagem, possuem um valor de mercado bem menor. Os resultados de profundidade de indentação na plastilina foram satisfatórios visto que atenderam a norma NIJ 0101.06 e utilizando a técnica de microscopia eletrônica de varredura, verificou-se que o compósito poliéster-juta capturou os fragmentos através do mecanismo de incrustação mecânica.

Palavras-chave: Blindagem multicamada, compósito poliéster-juta, ensaio balístico.

\section{FABRICATION OF POLYESTER MATRIX COMPOSITES REINFORCED WITH JUTE CLOTH APPLIED IN MULTILAYERED ARMOR}

\section{Abstract}

This paper presents some results obtained through ballistic tests on Multilayered Armor System (MAS). Following the specifications of American standard NIJ 0101.06, the tests were realized with ammunition $7.62 \times 51 \mathrm{~mm}$ that has a velocity above of $800 \mathrm{~m} / \mathrm{s}$. The MAS is composed of a front layer a hexagonal ceramic chip (alumina doped with $4 \%$ of nióbia), the second layer of polyester matrix composite reinforced with cloth of jute and the third layer an aluminum plate $5052 \mathrm{H} 34$. The utilization of polymeric composites reinforced with natural fibers to replace the aramid fibers is the big interest because they perform the same function in the armor system with the less expensive. The results of depth of indentation in clay witness were satisfactory because they have attended the standard NIJ 0101.06 and the scanning electron microscopy technique has showed that the polyester-jute composite has captured those fragments through of mechanism of mechanic incrustation..

Keywords: Multilayered armor, polyester-jute composite, ballistic tests.

1 Engenheiro Metalúrgico, mestre em Ciências dos Materiais, Instituto Militar de Engenharia, Rio de Janeiro, Rio de Janeiro - Brasil.

2 Engenheiro Metalúrgico, PhD em Materials Science And Engineering, Instituto Militar de Engenharia, Rio de Janeiro, Rio de Janeiro - Brasil.

3 Graduando em Engenharia, Instituto Militar de Engenharia, Rio de Janeiro, Rio de Janeiro - Brasil.

4 Engenheiro Metalúrgico, mestrando em Ciências dos Materiais, Instituto Militar de Engenharia, Rio de Janeiro, Rio de Janeiro - Brasil. 


\section{INTRODUÇÃO}

O aumento dos conflitos armados no Brasil, através de conflitos urbanos, e no mundo com guerras e atentados terroristas em conjunto das novas tecnologias de armas e munições impulsionam as pesquisas e desenvolvimentos por materiais que sejam capazes de resistir e proteger os seres humanos contra esses artefatos (1).

Os sistemas de blindagens multicamada (MAS - Multilayered Armor System) proporcionam uma proteção pessoal leve, efetiva e têm como objetivo não apenas absorver a energia do projétil, mas também de impedir a penetração de fragmentos. Um sistema de blindagem multicamada típico é composto por uma placa cerâmica, seguida por camadas de fibras de aramida apoiadas em uma placa de alumínio (2). O material cerâmico que possui elevada resistência à compressão, elevada dureza e baixa resistência à tração tem como função dissipar a maior parte da energia de impacto através da deformação, erosão e fragmentação do projetil $(2,3,4)$. Na segunda camada, normalmente, são utilizados materiais sintéticos: Kevlar ${ }^{\circledR}$ (fibras de aramida) ou Dyneema ${ }^{\circledR}$ (polietileno de ultra-alto peso molecular) que possuem baixa densidade, elevada resistência à tração. Essa camada tem como principal objetivo dissipar a energia de impacto através da absorção da energia cinética dos fragmentos gerados pelo impacto do projetil com a cerâmica. Em alguns casos, uma terceira camada pode ser adicionada no sistema de blindagem multicamada que é composta por um metal dúctil que tem a função de restringir a penetração do projétil $(1,2)$. De acordo com a norma americana NIJ 0101.06 (5), o sistema de blindagem multicamada tem o nível de proteção classificado como III, isto é, consegue resistir ao impacto de munição perfurante com calibre 7,62 × 51 milímetros, que possui velocidade de impacto superior a $800 \mathrm{~m} / \mathrm{s}$. O interesse em utilizar como segunda camada um material compósito de matriz polimérica reforçadas com fibras naturais como substituto das fibras sintéticas (fibras de Kevlar® ou Dyneema®) é pelo fato do material ter um custo menor, ser mais leve e tem o mesmo desempenho $(6,7,8)$. A utilização de compósitos reforçados com fibras naturais acarreta algumas vantagens, pois essas fibras são recursos alternativos, disponíveis em grande escala e possuem vantagens em relação às fibras sintéticas, tais como: biodegradabilidade, baixa densidade, natureza não tóxica, menor abrasividade nos equipamento que confeccionam os materiais, propriedades mecânicas aceitáveis e de baixo custo $(9,10)$. As fibras de juta são estudadas principalmente quando são utilizadas como material de reforço em matrizes poliméricas $(11,12)$. Este trabalho tem como objetivo analisar o desempenho balístico da blindagem multicamada contendo como camada intermediária o compósito de matriz poliéster reforçado com $30 \%$ em volume de tecido de juta utilizando ensaio de deformação na plastilina e comparar com o seu desempenho com o tecido de aramida e outros compósitos reforçados com fibras naturais. O sistema de blindagem multicamada é considerado eficiente se o trauma na plastilina for igual ou menor que 44 milímetros (5).

\section{MATERIAIS E MÉTODOS}

A fabricação da blindagem multicamada foi dividida em três etapas: a fabricação da pastilha cerâmica, preparação do compósito poliéster-juta e montagem da blindagem. Para a preparação do material cerâmico, primeira camada da blindagem, foi utilizada a alumina $\left(\mathrm{Al}_{2} \mathrm{O}_{3}\right)$ dopada com nióbia $\left(\mathrm{Nb}_{2} \mathrm{O}_{5}\right)$ na proporção na de $4 \%$ em massa com geometria hexagonal. Os pós de alumina, nióbia e o polietileno glicol, em fase líquida, foram devidamente pesados e em seguida acondicionados num 
moinho de bolas de alumina por $12 \mathrm{~h}$. Após este processo, o material foi deixado por $48 \mathrm{~h}$ na estufa a $80^{\circ} \mathrm{C}$ para secagem. Com o material seco, ocorreram às etapas de maceração e peneiramento para obtenção do pó e, em seguida, ocorreu à prensagem sob uma pressão de $60 \mathrm{MPa}$. Com isso, foram obtidos corpos verdes os quais foram levados ao forno para que fossem sinterizados. Na Figura 1 é mostrada uma pastilha cerâmica após a etapa de sinterização. No total foram produzidas oito pastilhas.

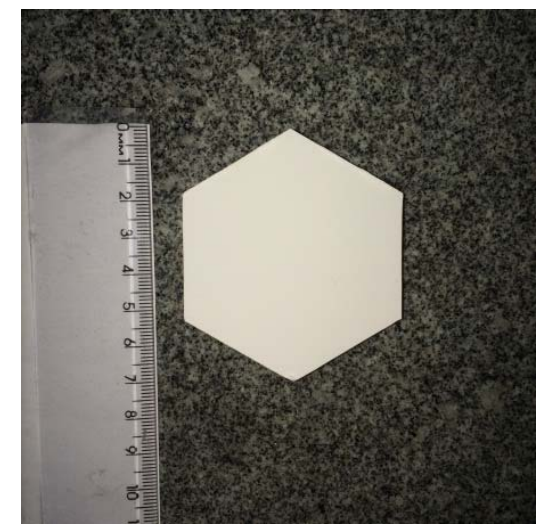

Figura 1. Pastilha cerâmica após a etapa de sinterização.

A confecção da placa de compósito, de dimensões $12,8 \times 15 \times 1 \mathrm{~cm}$, de matriz de poliéster reforçado com tecido de juta consistiu na prensagem em uma matriz metálica sob pressão de cinco toneladas, pelo período de vinte e quatro horas tempo de cura - adotando a proporção de $70 \%$ em volume de poliéster e $30 \%$ em volume de tecido onde estes foram cortados nas dimensões da matriz compondo várias camadas. Para a preparação da resina de poliéster (em estado líquido) foi adicionado, na proporção de $1 \%$ em peso, o catalisador peróxido metil-etil-cetona. Na Figura 2 é mostrada a placa do material compósito após a etapa de cura.

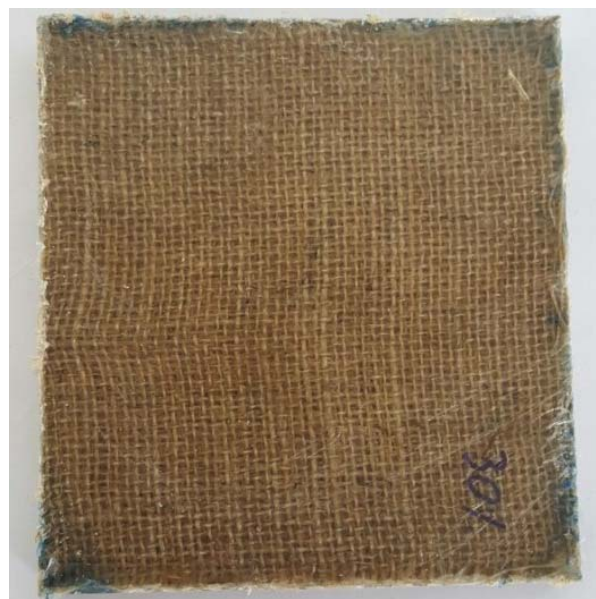

Figura 2. Placa do material compósito poliéster-juta.

A preparação dos corpos de prova multicamada foi realizada por meio da colagem das camadas de alumínio, do compósito e da cerâmica, utilizando cola de cura rápida ULTRAFLEX, que não interfere nos testes. Na Figura 3 é mostrado o esquema de montagem de uma blindagem multicamada e o corpo de prova final obtido. 

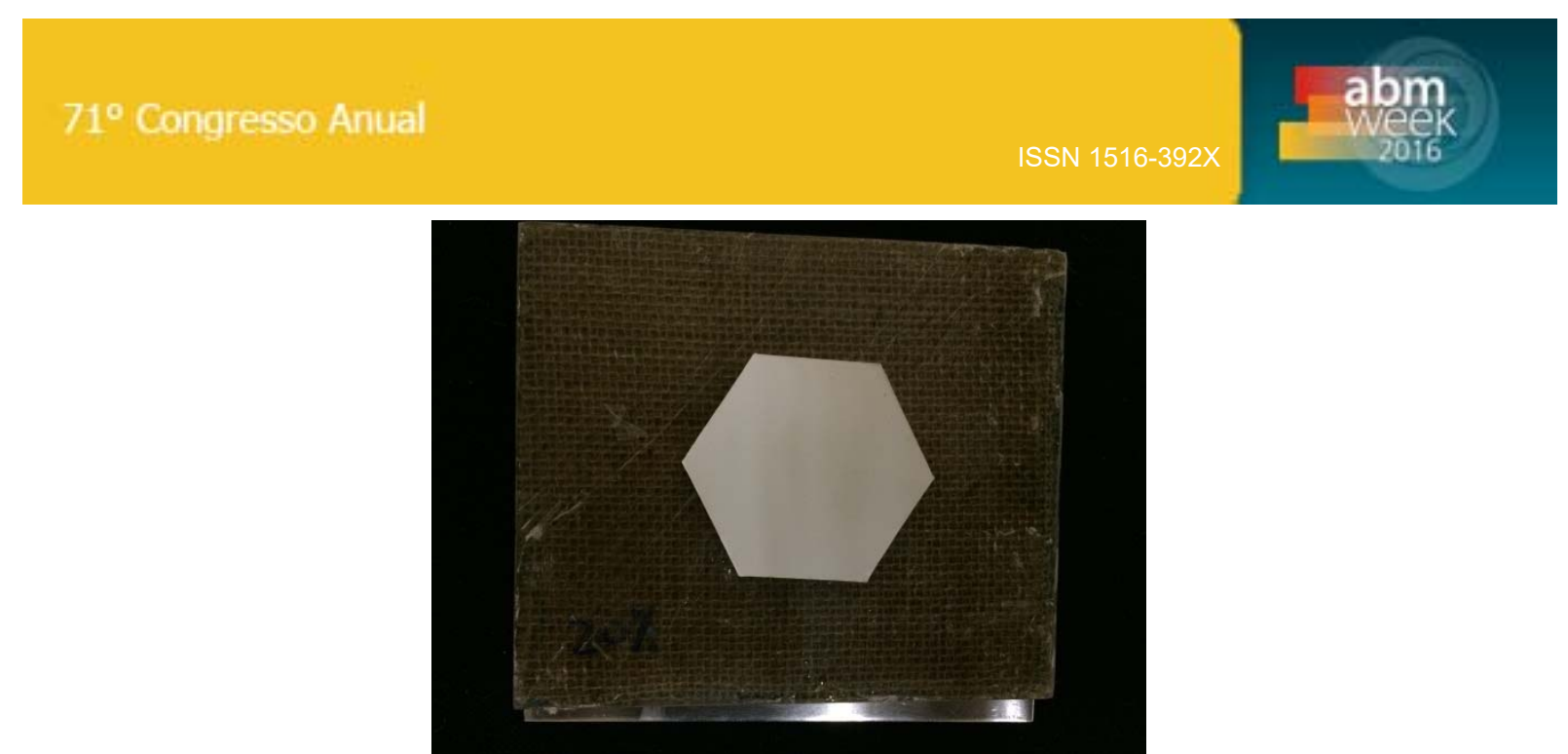

Figura 3. Corpo de prova final utilizado nos ensaios balísticos.

O ensaio balístico tem por finalidade verificar a capacidade de absorção de energia cinética resultante do impacto de um corpo em movimento. No referido trabalho foi observado à deformação na plastilina (massa que simula o corpo humano), provocada pelo disparo demonstrando, assim, o nível de proteção balística do sistema de blindagem. Na Figura 4 é mostrado o esquematicamente o sistema utilizado para a realização desses ensaios.

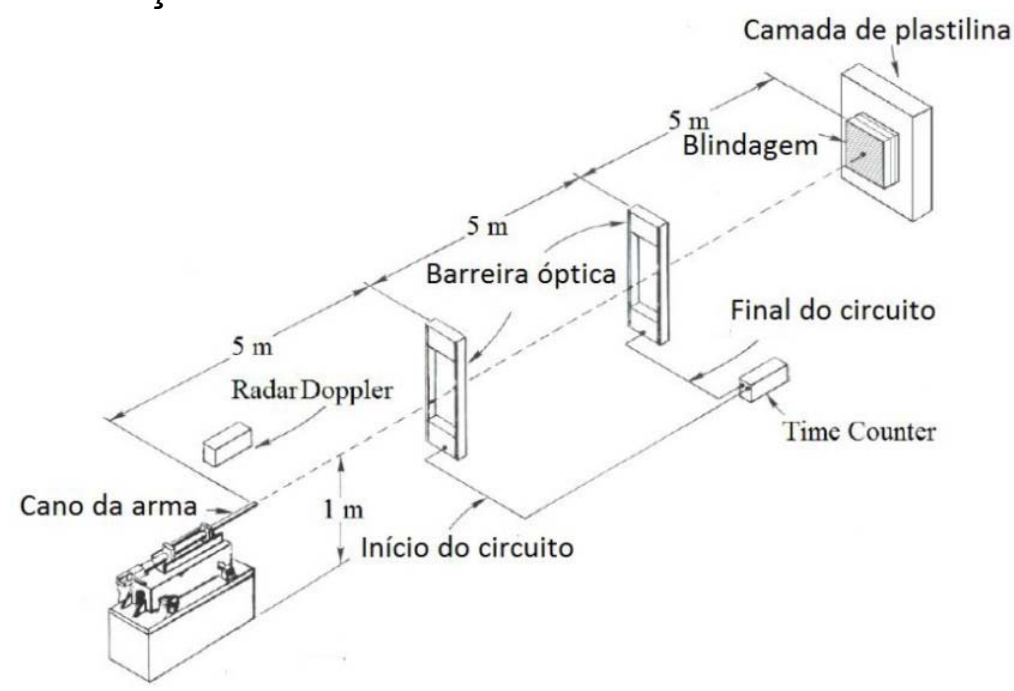

Figura 4. Esquema do sistema utilizado para os ensaios balísticos

Para a realização dos testes balísticos foram utilizadas as seguintes condições:

i) Munição: calibre 7,62 x $51 \mathrm{~mm} \mathrm{M1}$, conforme é fornecida comercialmente ao Exército; com projétil de massa de 9,7 gramas;

ii) Dispositivo: provete calibre 7,62 dotado de mira laser e um dispositivo de fixação do alvo.

iii) Distância do alvo: 15 metros, conforme previsto na norma ABNT NBR 15000 (13) para as munições 7,62 x $51 \mathrm{~mm}$;

iv) Ângulo de incidência no alvo: $90^{\circ}$;

v) Quantidade de tiros para cada situação específica: 8 (dez) disparos;

vi) Local: Centro de Avaliações do Exército (CAEx).

Os valores de indentação causadas na plastilina foram interpretados pelo método de Weibull, utilizando-se o programa Weibull Analysis. Após o ensaio balístico, com o objetivo de identificar os modos de falha dos materiais da blindagem multicamada, foi realizada avaliação microscópica dos fragmentos com o auxílio do microscópio eletrônico de varredura (MEV), utilizando elétrons secundários. 


\section{RESULTADOS E DISCUSSÃO}

Na Figura 5 é mostrado o sistema de blindagem fixado na plastilina antes e após o disparo da munição de calibre $7,62 \times 51 \mathrm{~mm}$, fazendo uma análise qualitativa é possível observar que o sistema de blindagem multicamada contendo como camada intermediária o compósito poliéster-juta resistiu ao impacto do projétil, já que o mesmo não perfurou a blindagem multicamada.
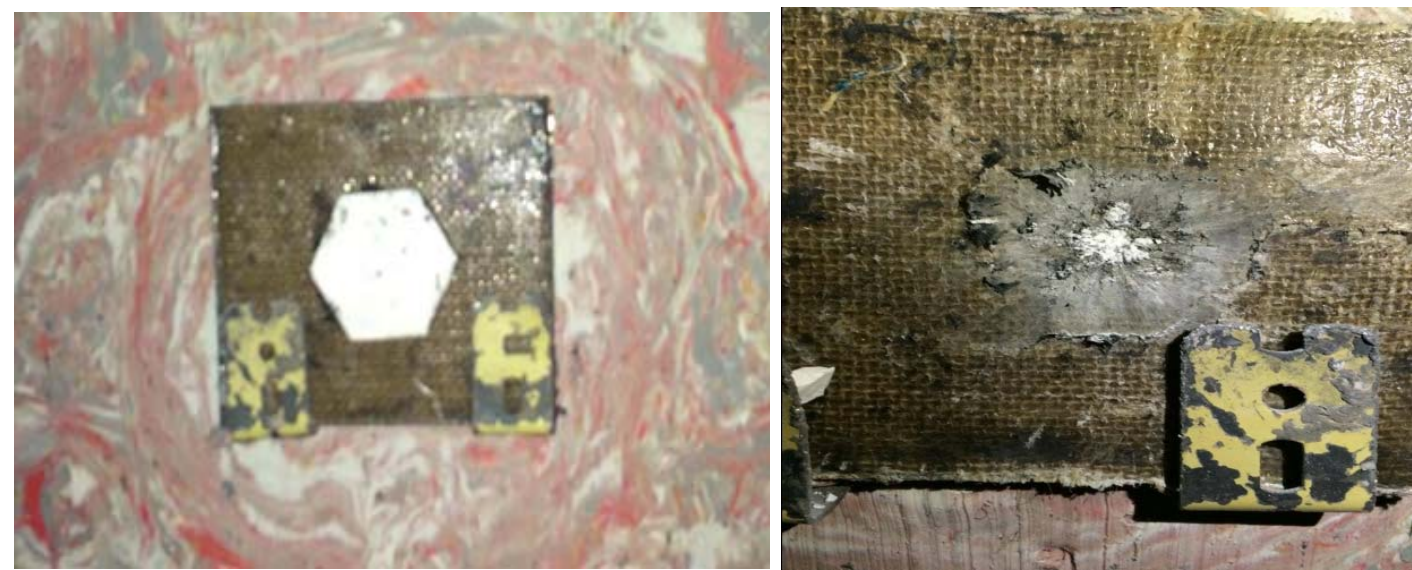

Figura 5. Sistema de blindagem multicamada: (a) antes e (b) após o ensaio balístico.

Os valores de indentação obtidos através do ensaio de deformação na plastilina foram tratados pela Estatística de Weibull, gerando o gráfico ilustrado na Figura 5, na Tabela 1 são apresentados os parâmetros de Weibull, acompanhados dos resultados estatísticos.

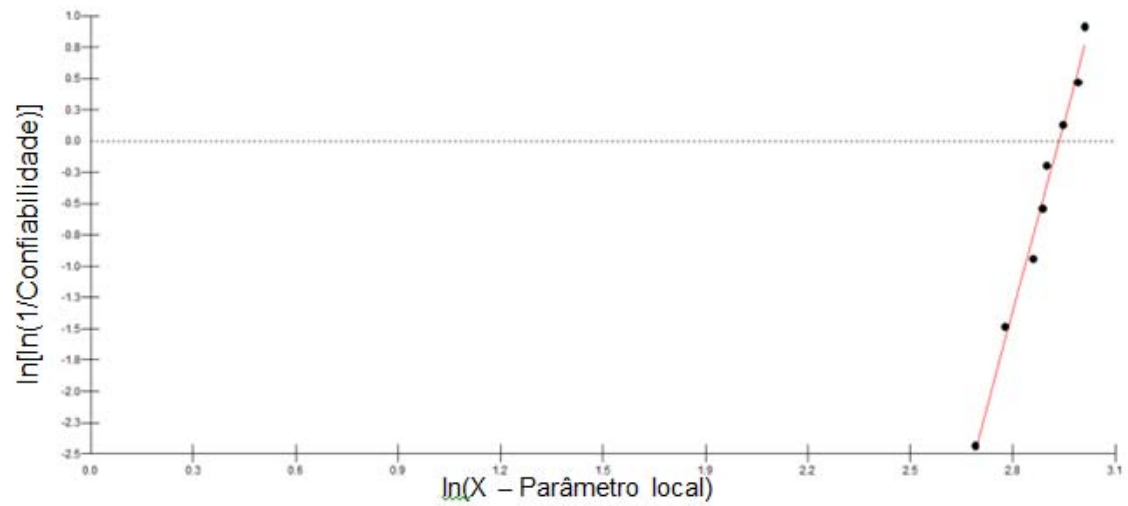

Figura 5. Gráfico da distribuição de Weibull da indentação para blindagem com poliéster-juta.

Tabela 1. Parâmetros de Weibull para blindagem multicamada com poliéster-juta

\begin{tabular}{cccccc}
\hline $\begin{array}{c}\text { Blindagem } \\
\text { Multicamada }\end{array}$ & $\begin{array}{c}\text { Módulo de } \\
\text { Weibull }(\boldsymbol{\beta})\end{array}$ & $\begin{array}{c}\text { Indentação } \\
\text { Característica } \\
(\boldsymbol{\theta})\end{array}$ & $\begin{array}{c}\text { Coeficiente de } \\
\text { Correlação }\left(\mathbf{R}^{2}\right)\end{array}$ & $\begin{array}{c}\text { Indentação } \\
\text { Média }(\mathbf{m m})\end{array}$ & $\begin{array}{c}\text { Desvio } \\
\text { Padrão (mm) }\end{array}$ \\
\hline Tecido-30\% & 9,76 & 18,73 & 0,9873 & 17,80 & 2,19 \\
\hline
\end{tabular}

O valor médio para profundidade indentação na plastilina foi de 17,80 milímetros, possuindo um menor valor que o estipulado pela norma NIJ 0101.06 que é de 44 milímetros. Os resultados obtidos através da Estatística de Weibull apontam uma boa homogeneidade (através do módulo de Weibull - $\beta$ ), obtendo uma baixa dispersão dos valores de indentação obtidos, ou seja, se tem uma boa confiabilidade dos resultados. O coeficiente de ajuste $\left(R^{2}\right)$, que varia de 0 a 1 , apresentou um valor 
de 0,9873 , o que aumenta a confiabilidade dos resultados, a Indentação característica mostrou um valor que melhor representa a indentação para o sistema de blindagem multicamada tendo como camada intermediária o compósito poliésterjuta.

$\mathrm{Na}$ Tabela 2 são mostrados os valores médios obtidos, através do ensaio de deformação na plastilina, do sistema de blindagem poliéster-juta e de sistemas de blindagem contendo outros materiais como camada intermediaria.

Tabela 2. Média dos valores obtidos nos ensaios balísticos.

\begin{tabular}{|c|c|c|c|c|c|c|}
\hline $\begin{array}{c}\text { Camada } \\
\text { Intermediária }\end{array}$ & $\begin{array}{c}\text { Espessura } \\
\text { Média } \\
(\mathrm{mm})\end{array}$ & $\begin{array}{c}\rho \\
\text { média } \\
\left(\mathrm{g} / \mathrm{cm}^{3}\right)\end{array}$ & $\begin{array}{l}\text { Indentação } \\
\text { Média (mm) }\end{array}$ & $\begin{array}{c}\text { Velocidade de } \\
\text { Impacto Média } \\
(\mathrm{m} / \mathrm{s})\end{array}$ & $\begin{array}{c}\text { Energia de } \\
\text { Impacto } \\
\text { Média (kJ) }\end{array}$ & Referências \\
\hline $\begin{array}{c}\text { Poliéster-Juta } \\
(30 \%)\end{array}$ & 10,61 & 1,17 & 17,80 & 853,08 & 3,53 & ${ }^{*} \mathrm{PT}$ \\
\hline $\begin{array}{c}\text { Epóxi-sisal } \\
(30 \%)\end{array}$ & 11,73 & 1,14 & 16,03 & 827,95 & 3,32 & (6) \\
\hline $\begin{array}{l}\text { Epóxi-Juta } \\
(30 \%)\end{array}$ & 11,42 & 1,17 & 20,67 & 833,81 & 3,37 & (8) \\
\hline Aramida & 10 & 1,40 & 22,67 & 836,81 & 3,41 & (8) \\
\hline
\end{tabular}

Como observado na tabela acima, o sistema de blindagem multicamada utilizando o tecido de aramida apresentou o maior valor de profundidade indentação, com valor de 22,67 milímetros, o que representa uma profundidade $27,36 \%$ maior que a indentação média da blindagem de compósito poliéster-juta. Essa diferença pode ser explicada pelo fato da densidade do tecido de aramida $\left(1,4 \mathrm{~g} / \mathrm{cm}^{3}\right)$ ser maior que o compósito poliéster-juta $\left(1,17 \mathrm{~g} / \mathrm{cm}^{3}\right)$, isso implica numa maior impedância de choque, fazendo com que o pulso compressivo transmitido para terceira camada (placa de alumínio) seja maior e, consequentemente, um maior valor na profundidade de indentação. Isso explica o motivo do sistema de blindagem contendo camada epóxi-sisal $\left(1,14 \mathrm{~g} / \mathrm{cm}^{3}\right)$ obter o menor valor de profundidade de indentação.

$\mathrm{Na}$ figura 6 são mostradas as imagens obtidas através do MEV do compósito poliéster-juta após a realização do ensaio balístico.

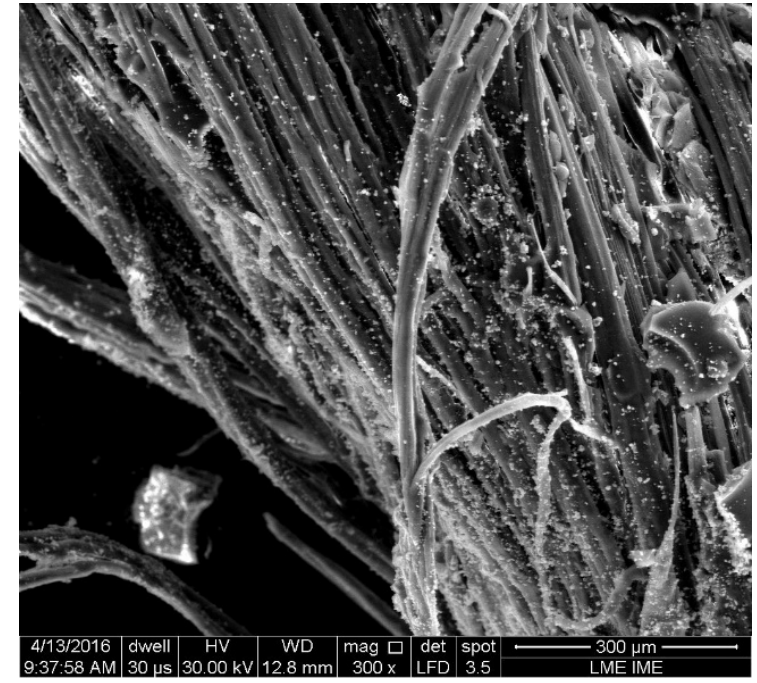

(a)

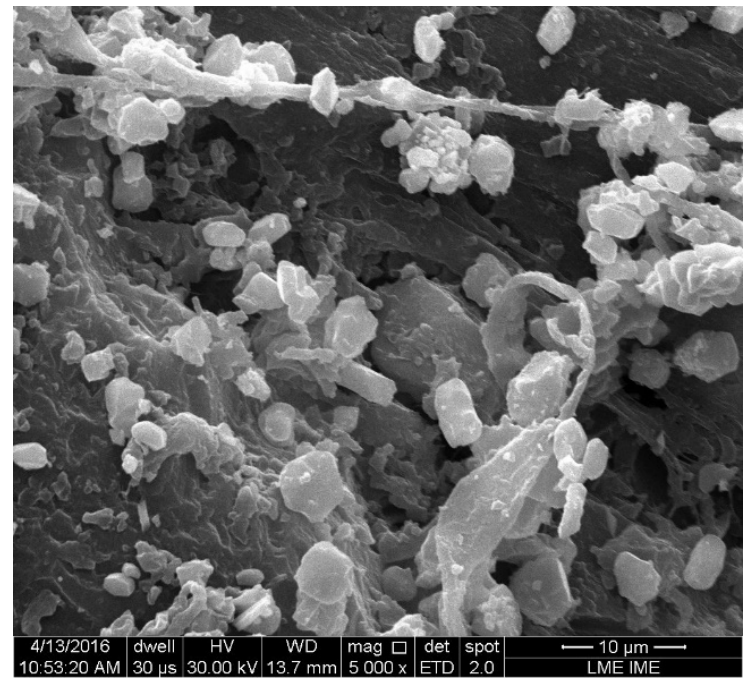

(b)

Figura 6. Superfície de fratura do compósito poliéster-juta: (a) 300x e (b) 5000x. 
Analisando as figuras acima, é possível observar que e o compósito poliéster-juta (Figura 6 (a)) ficou totalmente recoberta de partículas cerâmicas, indicando que o compósito absorveu a energia cinética dos fragmentos através do mecanismo de incrustação mecânica. Na Figura 6(b) é possível observar um empilhamento das partículas, devido à atração eletrostática e às forças que atuam na superfície do material, como a de Van der Walls, que possuem força o suficiente para manter as partículas incrustadas. Observa-se (Figura 6(b)), também, propagação de trinca na matriz de poliéster (fratura frágil) que também atua como dissipador de energia.

\section{CONCLUSÃO}

Os compósitos de matriz poliéster reforçada com tecido de juta utilizados como camada intermediária do sistema de blindagem multicamada atenderam as especificações da norma internacional NIJ 0101.06, ou seja, a profundidade de indentação foi menor que 44 milímetros. Esse sistema de blindagem apresentou uma menor profundidade indentação média $(17,80 \mathrm{~mm})$ em comparação a sistema de blindagem utilizando tecido de aramida $(22,67 \mathrm{~mm})$. Com auxilio das micrografias obtidas pelo MEV, foi possível observar que os compósitos (poliéster-juta) ensaiados tiveram suas superfícies recobertas por partículas cerâmicas, contribuindo pela dissipação de energia cinética através do mecanismo de incrustação mecânica.

\section{Agradecimentos}

A CAPES pelo incentivo financeiro

\section{REFERÊNCIAS}

1. WANG, L.; KANESALINGAM, S.; NAYAK, R.; PADHYE, R. Recent trends in Ballistic Protection. Textiles and Light Industrial Science and Technology (TLIST), Vol. 3. P. 3747. 2014.

2. MONTEIRO, S.N.; LIMA JR, E.P.; LOURO, L.H.L., SILVA, L.C.; DRELICH, J.W. Unlocking function of aramida fibers in multilayered ballistic armor. Accept for publication un Metallurgical and Materials Transactions A, November, 2014.

3. MEDVEDOVSKI, E. Ballistic Performance of Armour Ceramics: Influence of Design and Structure. Part 1. Ceramics International. Vol. 36, p. 2103-2115. 2010.

4. DA SILVA, M.V.; STAINER, D.; AL-QURESHI, H.A.; HOTZA, D. Blindagens Cerâmicas para Aplicações Balísticas: Uma Revisão. Cerâmica, Vol. 60, p. 323-331. 2014.

5. NIJ Standards-0101.06.Ballistic Resistance of Body Armor.U.S. Department of Justice/Office of Justice Programs - National Institute of Justice. 2008.

6. ARAÚJO, B.M. Avaliação do Comportamento Balístico de Blindagem Multicamada com Compósito de Epóxi Reforçado com Fibra de Sisal. Dissertação de Mestrado do Curso de Pós-Graduação em Ciência dos Materiais no Instituto Militar de Engenharia. 2015.

7. DA SILVA, L.C. Comportamento Balístico de Compósitos Epóxi-Fibra Natural em Blindagem Multicamada. Tese de Doutorado do Curso de Pós-Graduação em Ciência dos Materiais no Instituto Militar de Engenharia. 2014b.

8. DA LUZ, F.S. Avaliação do Comportamento Balístico de Blindagem Multicamada dom Compósito de Epóxi Reforçado com Fibra de Juta. Dissertação de Mestrado do Curso de Pós-Graduação em Ciência dos Materiais no Instituto Militar de Engenharia. 2014. 
9. SATYANARAYANA, K.G.; GUIMARÃES, J.L.; WYPYCH, F. Studies on lignocellulosic fibers of Brazil. Part I: Source, production, morphology, properties and applications. Composites: Part A, v. 38, p. 1694-1709, 2007.

10. MONTEIRO, S.N.; LOPES, F.P.D.; BARBOSA, A.P.B.; BEVITORI, A.B.; DA SILVA, I.L.A.; DA COSTA, L.L. Natural Lignocelullosic Fibers as Engineering Materials - An Overview. Metallurgical and Materials Transactions A, Vol. 42A, p. 2963-2974. 2011.

11. DA SILVA, I. L. A. Propriedades e Estruturas de Compósitos Poliméricos Reforçados com Fibras Contínuas de Juta. Tese de Doutorado do Curso de Pós-Graduação em Engenharia e Ciências de Materiais na Universidade Estadual do Norte Fluminense. 2014c.

12. WAMBUA, P.; VANGRIMDE, B.; LOMOV, S.; VERPOEST, I. The Response of Natural Fibre Composites to Ballistic Impact by Fragment Simulating Projectiles. Composite Structures, Vol. 77, p. 232-240. 2007.

13. ABNT NBR 15000. Blindagens para impactos balísticos - Classificação e critérios de avaliação, 2005. 